\title{
A Cumulative Residual Entropy Characterization of the Rayleigh Distribution and Related Goodness-of-Fit Test
}

\author{
S. Baratpour* and F. Khodadadi
}

Ferdowsi University of Mashhad

\begin{abstract}
Rayleigh distribution is widely used for life-time modeling and is important in electro vacuum devices and communication engineering. Rao et al. (2004) suggested the Cumulative Residual Entropy (CRE), which is the extension of the Shannon entropy to the the cumulative distribution function. In this paper, a general class of maximum CRE distributions is introduced and then we characterize the Rayleigh distribution and use it to construct a goodness-of-fit test for ascertaining appropriateness of such model. For constructing the test statistics, we use Cumulative residual Kullback-Leibler information (CKL) that was introduced by Baratpour and Habibi (2012). Critical values for various sample sizes determined by means of Monte Carlo simulations are presented for the test statistics. A Monte Carlo power analysis is performed for various alternatives and sample sizes in order to compare the proposed test with several existing goodness-of-fit tests based on the empirical distribution. We find that the proposed test has good power properties. The use of the proposed test is shown in an illustrative example.
\end{abstract}

Keywords. Cumulative residual entropy; maximum entropy; KullbackLeibler divergence; Rayleigh distribution; goodness of fit; power study.

MSC 2010: 62G10, 62E10, 94A17, 65C05.

\footnotetext{
* Corresponding author
} 


\section{Introduction}

The Rayleigh distribution is a special case of the Weibull distribution with shape parameter equal to 2 and a suitable model for life-testing studies. Also, the square root of an exponential random variable follows the Rayleigh distribution. Hence, by applying this transformation to the data, all Rayleighity tests can be utilized for the purpose of testing the goodness-of-fit to the exponential distribution. Polovko (1969), and Dyer and Whisenand (1973), demonstrated the importance of this distribution in electro vacum devices and communication engineering. Also, the Rayleigh distribution was originally derived in connection with a problem in acoustics, and has been used as the distance distribution between individuals in a spatial Poisson process. For more details on Rayleigh distribution the reader is referred to Johnson et al. (1994). The origin and other aspects of this distribution can be found in Siddiqui (1962), and Miller and Sackrowttz (1967). This model has the probability density function (pdf), cumulative distribution function (cdf), given respectively by

$$
f(x)=\frac{x}{\sigma^{2}} \exp \left(-\frac{x^{2}}{2 \sigma^{2}}\right), \quad x>0, \sigma>0,
$$

and

$$
F(x)=1-\exp \left(-\frac{x^{2}}{2 \sigma^{2}}\right), \quad x>0 .
$$

The concept of entropy is important for studies in many areas such as physics, probability and statistics, communication theory, and economics. An early definition of a measure of the entropy is the Shannon entropy (Shannon, 1948). In Shannons approach, discrete values and absolutely continuous distributions are treated in a somewhat different way through entropy and differential entropy, respectively. Shannon entropy is defined as

$$
H(X)=-\int_{-\infty}^{\infty} f(x) \ln f(x) d x,
$$

where $f$ is pdf if random variable $X$ is continuous, probability mass function if $X$ is discrete. Recent years have witnessed a growing interest in utilizing information-theoretic measures for distributional disparities as a tool for statistical inference in a variety of fields. For testing problems, the earliest work dates back to Vasicek (1976) which used Shannons maximum entropy to construct a goodness-of-fit test for normality. Vasicek's approach has 
much affected the development of entropy-based tests of fit for several parametric models; for example, see Grzegorzewski and Wieczorkowski (1999), Alizadeh Noughabi and Arghami (2011) and Taufer (2002). In probability theory and information theory, the Kullback-Leibler divergence (Kullback and Leibler, 1951; Kullback, 1959,1987) is a non-symmetric measure of the difference between two probability distributions $P$ and $Q$. Specifically, the Kullback-Leibler divergence of $Q$ from $P$ is a measure of the information lost when $Q$ is used to approximate $P$. For discrete probability distributions $P$ and $Q$, the KL divergence of $Q$ from $P$ is defined to be

$$
\mathrm{KL}(P: Q)=\sum_{i} \ln \frac{P(i)}{Q(i)} P(i) .
$$

For distributions $F$ and $G$ of a continuous random variable, KL is defined to be

$$
\mathrm{KL}(F: G)=\int_{-\infty}^{\infty} \ln \left\{\frac{f(x)}{g(x)}\right\} f(x) d x,
$$

where $f$ and $g$ denote the pdfs of $F$ and $G$, respectively. Tests of fit based on Kullback-Leibler information have been developed; see Ebrahimi et al. (1992), Choi et al. (2004) and Gurevich and Davidson (2008).

Considering the complementary cumulative distribution function instead of the probability density function in the definition of Shannon entropy leads to a new entropy measure named cumulative residual entropy (CRE) (Rao et al., 2004).

$\mathrm{CRE}$ is defined as

$$
\operatorname{CRE}(X)=-\int_{R_{+}^{N}} P(|X|>u) \ln P(|X|>u) d u
$$

where $N$ is the dimension of the random vector $X$. In reliability theory, CRE is based on survival function, $\bar{F}(x)=1-F(x)$, and is defined as

$$
\mathrm{CRE}(\mathrm{X})=-\int_{0}^{\infty} \bar{F}(x) \ln \bar{F}(x) d x .
$$

Clearly, this definition is valid both for a discrete or an absolutely continuous random variable. In addition, unlike Shannon entropy it is always positive, while preserving many interesting properties of Shannon entropy. The concept of CRE has found nice interpretations and applications in the fields of reliability, see Asadi and Zohrevand (2007) where the concept of 
dynamic CRE was introduced. Wang et al. (2003) defined a new matching criterion based on CRE for application to the image alignment problem and compared it to methods that use the Shannon entropy in defining a match measure images alignment (see, Wang and Vermuri, 2007). Di Crescenzo and Longobardi (2009) showed that the cumulative entropy of a random lifetime $X$ can be expressed as the expectation of its mean inactivity time evaluated at $t$. Baratpour (2010) characterized the first order statistics based on the CRE. Baratpour and Habibi (2012) developed a consistent test for testing the hypothesis of exponentiality against some alternatives.

The following example will be used in Section 2 .

Example 1. For r.v. $X$ with Rayleigh $(\sigma)$ distribution, we have

$$
E(X)=\sigma \sqrt{\frac{\pi}{2}}, \quad E\left(X^{3}\right)=3 \sigma^{3} \sqrt{\frac{\pi}{2}},
$$

and

$$
\begin{aligned}
\operatorname{CRE}(X) & =-\int_{0}^{\infty} \exp \left(-\frac{x^{2}}{2 \sigma^{2}}\right) \ln \left\{\exp \left(-\frac{x^{2}}{2 \sigma^{2}}\right)\right\} d x \\
& =\frac{1}{2 \sigma^{2}} \int_{0}^{\infty} x^{2} \exp \left(-\frac{x^{2}}{2 \sigma^{2}}\right) d x \\
& =\frac{1}{2} \int_{0}^{\infty} \exp \left(-\frac{x^{2}}{2 \sigma^{2}}\right) d x \\
& =\sigma \frac{\sqrt{2 \pi}}{4}
\end{aligned}
$$

This article is organized as follows. In Section 2, we introduce a general distribution which maximizes the CRE within a special class of distributions and then characterize the rayleigh distribution in a class of distributions with some moment constraint. In Section 3, we construct a consistent test for testing the hypothesis of Rayleighity against some alternatives. This test is based on a new measure of distance between two distributions that was defined by Baratpour and Habibi (2012). In Section 4, we consider some power estimates obtained by the method of Monte Carlo simulation. An illustrative example for implementing the proposed test is provided in Section 5 . 


\section{A CRE Characterization}

In this section, a general class of maximum CRE distributions is introduced and then we characterize the Rayleigh low as the distribution with the maximum CRE among all absolutely continuous distributions with support $(0, \infty)$ and given first and third moment.

Theorem 1. The random variable $X$ with distribution function

$$
F(x ; \lambda)=1-\exp \left(-\sum_{i=1}^{k} \lambda_{i} x^{i}\right), \quad \lambda_{i} \geqslant 0
$$

has maximum CRE within class $\boldsymbol{A}$ of absolutely continuous random variables $Y$ with support $(0, \infty)$, which satisfy

$$
\sum_{i=0}^{k} \frac{\lambda_{i}}{i+1} E\left(X^{i+1}\right)=\sum_{i=0}^{k} \frac{\lambda_{i}}{i+1} E\left(Y^{i+1}\right),
$$

where $\lambda_{0}=-1$.

Proof. Let random variable $Y$ be a member of $\mathbf{A}$. By log-sum inequality and inequality $y \ln \frac{y}{x} \geqslant y-x, x>0, y>0$, we conclude that

$$
\begin{aligned}
\int_{0}^{\infty} P(Y>t) \ln \frac{P(Y>t)}{P(X>t)} d t & \geqslant E(Y) \ln \frac{E(Y)}{E(X)} \\
& \geqslant E(Y)-E(X) .
\end{aligned}
$$

Thus,

$$
\begin{aligned}
\int_{0}^{\infty} P(Y>t) \ln P(Y>t) d t & \geqslant \int_{0}^{\infty} P(Y>t) \ln P(X>t) d t+E(Y)-E(X) \\
& =-\sum_{i=0}^{k} \lambda_{i} \int_{0}^{\infty} P(Y>t) t^{i} d t+E(Y)-E(X) .
\end{aligned}
$$


By noting that $\int_{0}^{\infty} P(Y>t) t^{i} d t=\frac{E\left(Y^{i+1}\right)}{i+1}$ and $(1)$, we have

$$
\begin{aligned}
\operatorname{CRE}(Y) & \leqslant \sum_{i=1}^{k} \frac{\lambda_{i}}{i+1} E\left(Y^{i+1}\right)-E(Y)+E(X) \\
& =\sum_{i=0}^{k} \frac{\lambda_{i}}{i+1} E\left(Y^{i+1}\right)+E(X) \\
& =\sum_{i=0}^{k} \frac{\lambda_{i}}{i+1} E\left(X^{i+1}\right)+E(X) \\
& =\sum_{i=1}^{k} \frac{\lambda_{i}}{i+1} E\left(X^{i+1}\right)
\end{aligned}
$$

It can be shown that $\operatorname{CRE}(X)=\sum_{i=1}^{k} \frac{\lambda_{i}}{i+1} E\left(X^{i+1}\right)$. Thus, the proof is completed.

By Theorem 1, we conclude the following Corollary.

Corollary 1. (i) The exponential distribution with parameter $\lambda$ maximizes the CRE among all distributions with support $(0, \infty)$ subject to constraint $\frac{E\left(X^{2}\right)}{2 E(X)}$, which is the result presented in Rao et al. (2004).

(ii) The Rayleigh distribution with parameter $\sigma$ maximizes the CRE among all absolutely continuous distributions with support $(0, \infty)$ subject to restriction $E\left(X^{3}\right)-6 \sigma^{2} E(X)+3 \sigma^{3} \sqrt{\frac{\pi}{2}}=0$.

By Corollary 1 (ii), we conclude the following Corollary.

Corollary 2. The r.v. X with Rayleigh $(\sigma)$ distribution is characterized by the property that $X$ attains maximum CRE among all nonnegative, absolutely continuous r.v.s $Y$ subject to restrictions $E(Y)=\nu$ and $E\left(Y^{3}\right)=\omega$, where $\sigma^{2}=\frac{\omega}{3 \nu}$. 


\section{Cumulative Residual Entropy-Based Test}

Let $X_{1}, \ldots, X_{n}$ be nonnegative; independent and identically distributed (iid) random variables from a continuous distribution function $F$ with order statistics, $X_{(1)} \leqslant \ldots \leqslant X_{(n)}$, and with finite $E\left(X_{1}\right)=\sigma \sqrt{\frac{\pi}{2}}$ and $E\left(X_{1}^{3}\right)=3 \sigma^{3} \sqrt{\frac{\pi}{2}}$ $\left(\frac{E\left(X_{1}^{3}\right)}{3 E\left(X_{1}\right)}=\sigma^{2}\right)$. Let $F_{0}(x)=1-\exp \left(-\frac{x^{2}}{2 \sigma^{2}}\right)$ denote a Rayleigh distribution function, where $\sigma$ is the unknown parameter. The aim of this article is testing the hypothesis

$$
H_{0}: F(x)=F_{0}(x, \sigma), \quad \text { versus } \quad H_{a}: F(x) \neq F_{0}(x, \sigma) .
$$

Baratpour and Habibi (2012) defined a new measure of distance between two distribution based on CRE called cumulative Kullback-Leibler (CKL) divergence and construct a goodness-of-fit test for exponentiality. They proved that CKL is non-negative and equality holds if and only if $F=G$, a.e. This measure is defined as follows

Defenition 1. If $X$ and $Y$ be two nonnegative random variables with respectively distribution functions $F$ and $G$, then CKL between these distributions is defined as

$$
\operatorname{CKL}(F: G)=\int_{0}^{\infty} \bar{F}(x) \ln \frac{\bar{F}(x)}{\bar{G}(x)} d x-\{E(X)-E(Y)\},
$$

where $\bar{F}(x)=1-F(x)$ and $\bar{G}(x)=1-G(x)$ are respectively cumulative residual distribution of $X$ and $Y$.

Thus, under the null hypothesis, $\operatorname{CKL}\left(F: F_{0}\right)=0$ and large value of $\operatorname{CKL}\left(F ; F_{0}\right)$ leads us to reject the null hypothesis $H_{0}$ in favor of the alternative hypothesis $H_{a}$. Since evaluation of the integral in $\operatorname{CKL}\left(F: F_{0}\right)$ requires complete knowledge of $F$ and $F_{0}$, then $\operatorname{CKL}\left(F: F_{0}\right)$ is not operational. We operationalize $\operatorname{CKL}\left(F: F_{0}\right)$ by developing a discrimination information statistics. With this in mind, $\operatorname{CKL}\left(F ; F_{0}\right)$ is written as

$$
\begin{aligned}
\operatorname{CKL}\left(F: F_{0}\right) & =-\operatorname{CRE}(F)-\int_{0}^{\infty} \bar{F}(x) \ln \bar{F}_{0}(x ; \sigma) d x-E(X)+\sigma \sqrt{\frac{\pi}{2}} \\
& =-\operatorname{CRE}(F)+\frac{1}{2 \sigma^{2}} \int_{0}^{\infty} x^{2} \bar{F}(x) d x-E(X)+\sigma \sqrt{\frac{\pi}{2}} .
\end{aligned}
$$


An estimator of $\operatorname{CKL}\left(F: F_{0}\right)$ is obtained by replacing in $(2), F$ by empirical distribution function $F_{n}(x)=\sum_{i=0}^{n-1} \frac{i}{n} I_{\left[x_{(i)}, x_{(i+1)}\right]}(x)$ and replacing $\sigma$ and $E(X)$ by $\sqrt{\frac{\sum_{i=1}^{n} X_{i}^{3}}{3 \sum_{i=1}^{n} X_{i}}}$ and $\bar{X}=\frac{1}{n} \sum_{i=1}^{n} X_{i}$, respectively. Thus

$$
\widehat{\operatorname{CKL}}\left(F: F_{0}\right)=-\operatorname{CRE}\left(F_{n}\right)-\frac{1}{2} \bar{X}+\sqrt{\frac{\pi}{2}} \cdot \sqrt{\frac{\sum_{i=1}^{n} X_{i}^{3}}{3 \sum_{i=1}^{n} X_{i}}},
$$

where $\operatorname{CRE}\left(F_{n}\right)=-\sum_{i=1}^{n-1} \frac{n-i}{n}\left(\ln \frac{n-i}{n}\right)\left\{X_{(i+1)}-X_{(i)}\right\}$. Because the test statistics must dont depend on parameter, By dividing (3) to $\bar{X}$, the test statistics is defined as

$$
C K_{n}=\frac{\sum_{i=1}^{n-1} \frac{n-i}{n}\left(\ln \frac{n-i}{n}\right)\left\{X_{(i+1)}-X_{(i)}\right\}+\sqrt{\frac{\pi}{2}} \sqrt{\frac{\sum_{i=1}^{n} X_{i}^{3}}{3 \sum_{i=1}^{n} X_{i}}}}{\bar{X}}
$$

We reject $H_{0}$ at the significance level $\alpha$ and favor $H_{a}$ if $\mathrm{CK}_{n} \geqslant \mathrm{CK}_{n, 1-\alpha}$, where $\mathrm{CK}_{n, 1-\alpha}$ is $100(1-\alpha)$ - precentile of $C K_{n}$ under $H_{0}$.

Rao, et al. (2004) proved that $\operatorname{CRE}\left(F_{n}\right) \rightarrow \operatorname{CRE}(F)$ a.s. Thus $\operatorname{CRE}\left(F_{n}\right)$ is a consistent estimator for $\operatorname{CRE}(F)$. By consistency of $\frac{\sum_{i=1}^{n} X_{i}^{3}}{3 \sum_{i=1}^{n} X_{i}}$ for $\sigma^{2}$ and apply-

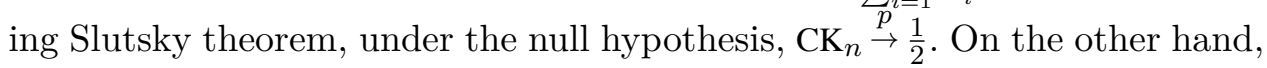
by Corollary 2 the Rayleigh distribution maximizes CRE among all distributions that have the same $E(X)$ and $E\left(X^{3}\right)$, so $\operatorname{CRE}(F)<\operatorname{CRE}\left(F_{0}\right)=\frac{\sqrt{2 \pi}}{4} \sigma$. Under $H_{a}, \operatorname{CRE}\left(F_{n}\right) \rightarrow \operatorname{CRE}(F)$ a.s; thus $\mathrm{CK}_{n} \stackrel{p}{\rightarrow} \frac{-\operatorname{CRE}(F)+\sqrt{\frac{\pi}{2}} \sigma}{\sqrt{\frac{\pi}{2}} \sigma}>\frac{-\operatorname{CRE}\left(F_{0}\right)+\sqrt{\frac{\pi}{2}} \sigma}{\sqrt{\frac{\pi}{2}} \sigma}$ $=\frac{1}{2}$. This means that the $\mathrm{CK}_{n}$ test is a consistent test.

\section{Monte Carlo Results}

In this section, we obtain the percentage points of the proposed test and study its power by Mont Carlo simulation.

\subsection{Critical Values of the Test Statistics}

The distribution of $\mathrm{CK}_{n}$ under the null hypothesis has not been obtained analytically. To determine the percentage points $\mathrm{CK}_{n, 1-\alpha}$, Mont Carlo simulations were employed.

In order to obtain the percentiles of the null distribution of $\mathrm{CK}_{n}, 100,000$ 
samples of size $n$ were generated from the Rayleigh distribution with $\sigma=2$, for selected values $n=1, \ldots, 39$ and 40 to 60 by 5 , and 70 to 100 by 10 . For each sample, the $\mathrm{CK}_{n}$ statistics as defined in (4) was calculated. The values were then used to determine the critical values $\mathbf{C K}_{n, 0.95}$ and $\mathbf{C K}_{n, 0.99}$. A selection of the 95 and 99 percent points is presented in Table 1.

The type I error control using the 0.95 percentiles of the $\mathrm{CK}_{n}$ statistics was evaluated by simulating random samples from a spectrum of Rayleigh populations. A selection of the result is presented in Table 2. It can be seen that the empirical percentiles given in Table 2 provide an excellent type I error control.

Table 1. Critical values of the test statistic $\mathrm{CK}_{n}$

\begin{tabular}{ccccccc}
\hline \hline & \multicolumn{2}{c}{$\mathrm{CK}_{n}$} & & \multicolumn{2}{c}{$\mathrm{CK}_{n}$} \\
\cline { 2 - 3 } \cline { 6 - 7 } & $\alpha=0.01$ & $\alpha=0.05$ & & $\alpha=0.01$ & $\alpha=0.05$ \\
\hline 1 & 0.7236013 & 0.7236013 & & 25 & 0.5647094 & 0.5421782 \\
2 & 0.7214782 & 0.7093749 & & 26 & 0.5636395 & 0.5407059 \\
3 & 0.7175996 & 0.6734160 & & 27 & 0.5614636 & 0.5397082 \\
4 & 0.7017060 & 0.6465053 & & 28 & 0.5593608 & 0.5384825 \\
5 & 0.6850819 & 0.5606804 & & 29 & 0.5575642 & 0.5373767 \\
6 & 0.6681757 & 0.6151675 & & 30 & 0.5570241 & 0.5366245 \\
7 & 0.6534651 & 0.6043387 & & 31 & 0.5557888 & 0.5356270 \\
8 & 0.6425021 & 0.5954569 & & 32 & 0.5536211 & 0.5345317 \\
9 & 0.6323886 & 0.5885379 & & 33 & 0.5527510 & 0.5338593 \\
10 & 0.6269256 & 0.5826478 & & 34 & 0.5508095 & 0.5329456 \\
11 & 0.6166661 & 0.5769818 & & 35 & 0.5505608 & 0.5325437 \\
12 & 0.6094163 & 0.5718854 & & 36 & 0.5489008 & 0.5316753 \\
13 & 0.6047603 & 0.5678996 & & 37 & 0.5481700 & 0.5309661 \\
14 & 0.5986721 & 0.5641181 & & 38 & 0.5474240 & 0.5303068 \\
15 & 0.5962232 & 0.5616213 & & 39 & 0.5462676 & 0.5295431 \\
16 & 0.5902378 & 0.5585082 & & 40 & 0.5455303 & 0.5291758 \\
17 & 0.5879517 & 0.5563432 & & 45 & 0.5412851 & 0.5265232 \\
18 & 0.5838778 & 0.5539128 & & 50 & 0.5376516 & 0.5243472 \\
19 & 0.5811981 & 0.5517890 & & 55 & 0.5354097 & 0.5226402 \\
20 & 0.5774558 & 0.5500101 & & 60 & 0.5325251 & 0.5210588 \\
21 & 0.5739829 & 0.5477154 & & 70 & 0.5281727 & 0.5184013 \\
22 & 0.5716899 & 0.5464284 & & 80 & 0.5256438 & 0.5166467 \\
23 & 0.5698107 & 0.5447773 & & 90 & 0.5231150 & 0.5150180 \\
24 & 0.5669811 & 0.5432045 & & 100 & 0.5211445 & 0.5136784 \\
\hline & & & & &
\end{tabular}


Table 2. Type I error control of $\mathrm{CK}_{n}$ test: $\alpha=0.05$ (Simulation estimates based on 100,000 replications)

\begin{tabular}{cccc}
\hline \hline Rayleigh $(\sigma)$ & $n=5$ & $n=15$ & $n=25$ \\
\hline$\sigma=3$ & 0.05110 & 0.05018 & 0.04941 \\
$\sigma=4$ & 0.04802 & 0.04987 & 0.04946 \\
$\sigma=5$ & 0.04960 & 0.04942 & 0.04992 \\
$\sigma=6$ & 0.05030 & 0.05078 & 0.05027 \\
\hline
\end{tabular}

\subsection{Power Study}

In this subsection, the power of the $\mathrm{CK}_{n}$ test is estimated against several alternative. The method is that of Monte Carlo simulation of the distribution of $\mathrm{CK}_{n}$ under alternative distributions. For each alternative, 100000 samples of sizes $n=5,10,15,20,25,30,35,40,50,60$ were generated, and the test power is estimated by the frequency of the samples falling into the critical region. The continuous alternative investigated were gamma distributions with density function

$$
f(x ; \lambda, \beta)=\frac{x^{\beta-1} \exp \left(-\frac{x}{\lambda}\right)}{\lambda^{\beta} \Gamma(\beta)}, \quad \beta>0, \lambda>0, x>0
$$

log-normal distribution with density function

$$
\begin{aligned}
f(x ; \mu, \nu)=\frac{1}{x \nu \sqrt{ }(2 \pi)} \exp \left\{-\frac{1}{2 \nu^{2}}(\ln x-\mu)^{2}\right\} & \\
& -\infty<\mu<\infty, \nu>0, x>0 ;
\end{aligned}
$$

inverse Gaussian distribution with density function

$$
f(x ; \mu, \lambda)=\sqrt{\frac{\lambda}{2 \pi x^{3}}} \exp \left\{-\frac{\lambda(x-\mu)^{2}}{2 x \mu^{2}}\right\}, \quad \mu>0, \lambda>0, x>0 .
$$

The power of the $\mathrm{CK}_{n}$ test is compared to that of some other tests for Rayleighity against the same alternative. These selected tests are VanSoest test (Van-Soest, 1969), Finkelstein and Schafers test (Finkelstein and Schafer, 1971) and Anderson-Darling (Anderson and Darling, 1954). These 
test statistics are respectively as follows

$$
\begin{aligned}
& W^{2}=\sum_{i=1}^{n}\left\{F_{0}\left(X_{(i)} ; \widehat{\sigma}\right)-\frac{2 i-1}{2 n}\right\}^{2}+\frac{1}{12 n} \\
& =\sum_{i=1}^{n}\left[1-\exp \left\{-\frac{\pi n^{2} x_{i}^{2}}{4\left(\sum_{1}^{n} x_{i}\right)^{2}}\right\}-\frac{2 i-1}{2 n}\right]^{2}+\frac{1}{12 n}, \\
& S^{*}=\sum_{i=1}^{n} \max \left\{\left|F_{0}\left(X_{(i)} ; \widehat{\sigma}\right)-\frac{i}{n}\right|,\left|F_{0}\left(X_{(i)} ; \widehat{\sigma}\right)-\frac{i-1}{n}\right|\right\} \\
& =\sum_{i=1}^{n} \max \left[\left|1-\exp \left\{-\frac{\pi n^{2} x_{i}^{2}}{4\left(\sum_{1}^{n} x_{i}\right)^{2}}\right\}-\frac{i}{n}\right|\right. \text {, } \\
& \left.\left|1-\exp \left\{-\frac{\pi n^{2} x_{i}^{2}}{4\left(\sum_{1}^{n} x_{i}\right)^{2}}\right\}-\frac{i-1}{n}\right|\right] \text {, } \\
& A^{2}=-n-\sum_{i=1}^{n} \frac{2 i-1}{n}\left\{\ln \left(F_{0}\left(X_{(i)} ; \widehat{\sigma}\right)\right\}+\ln \left\{1-F_{0}\left(X_{(n+1-i)} ; \widehat{\sigma}\right)\right\}\right. \\
& =-n-\sum_{i=1}^{n} \frac{2 i-1}{n}\left(\ln \left[1-\exp \left\{-\frac{\pi n^{2} x_{i}^{2}}{4\left(\sum_{1}^{n} x_{i}\right)^{2}}\right\}\right]-\frac{\pi n^{2} x_{n+1-i}^{2}}{4\left(\sum_{1}^{n} x_{i}\right)^{2}}\right)
\end{aligned}
$$

where $\sigma$ has been substituted by $\widehat{\sigma}=\frac{\sum_{i=1}^{n} X_{i}}{n \sqrt{\frac{\pi}{2}}} . H_{0}$ is rejected of large values of $W_{2}, S^{*}$ and $A^{2}$.

Tables 3-5 list power estimates of 0.1 and 0.5 size test with various sample size $n$. It is apparent from these table that the $\mathrm{CK}_{n}$ test appears to be more powerful than $W^{2}, S^{*}$ and $A^{2}$ testes. On the other-hand, compared with all the test proposed, $\mathrm{CK}_{n}$ test is moderately easy to compute. These results, together with the asymptotic properties of $\mathrm{CK}_{n}$, suggest that the $\mathrm{CK}_{n}$ test may be preferred in many situations. It is also remarkable that the power of the all tests against any alternative shows an increasing pattern for the sample size. 
Table 3. Power comparison for the tests $\mathrm{CK}_{n}, W^{2}$ and $S^{*}$ when the alternative distribution is gamma $(\lambda, \beta)$, where $\lambda=1,2$ and $\frac{E\left(X_{1}^{3}\right)}{3 E\left(X_{1}\right)}=4$ at the significance levels $\alpha=0.01$ and $\alpha=0.05$, and sample sizes $n=5,10,15,20,25,30,35,40,50,60$.

\begin{tabular}{|c|c|c|c|c|c|c|}
\hline$n$ & $\lambda$ & $\alpha$ & $\mathrm{CK}_{n}$ & $W^{2}$ & $S^{*}$ & $A^{2}$ \\
\hline \multirow[t]{4}{*}{5} & 1 & 0.01 & 0.26789 & 0.17514 & 0.17438 & 0.36098 \\
\hline & & 0.05 & 0.75408 & 0.30573 & 0.31268 & 0.52386 \\
\hline & 2 & 0.01 & 0.07112 & 0.03386 & 0.03111 & 0.06722 \\
\hline & & 0.05 & 0.14274 & 0.09630 & 0.09432 & 0.16101 \\
\hline \multirow[t]{4}{*}{10} & 1 & 0.01 & 0.48909 & 0.39369 & 0.38890 & 0.63051 \\
\hline & & 0.05 & 0.65003 & 0.57509 & 0.57495 & 0.77149 \\
\hline & 2 & 0.01 & 0.13519 & 0.06557 & 0.06002 & 0.11813 \\
\hline & & 0.05 & 0.24550 & 0.16285 & 0.15571 & 0.2480 \\
\hline \multirow[t]{4}{*}{15} & 1 & 0.01 & 0.65614 & 0.59188 & 0.58405 & 0.79506 \\
\hline & & 0.05 & 0.80082 & 0.75806 & 0.75365 & 0.89353 \\
\hline & 2 & 0.01 & 0.19843 & 0.10273 & 0.09810 & 0.16802 \\
\hline & & 0.05 & 0.33860 & 0.22763 & 0.22268 & 0.32547 \\
\hline \multirow[t]{4}{*}{20} & 1 & 0.01 & 0.77806 & 0.70486 & 0.72788 & 0.89446 \\
\hline & & 0.05 & 0.88965 & 0.86581 & 0.863620 & .95183 \\
\hline & 2 & 0.01 & 0.25834 & 0.14380 & 0.13396 & 0.21965 \\
\hline & & 0.05 & 0.40989 & 0.29184 & 0.28643 & 0.3924 \\
\hline \multirow[t]{4}{*}{25} & 1 & 0.01 & 0.8636 & 0.83821 & 0.83518 & 0.94742 \\
\hline & & 0.05 & 0.94085 & 0.93013 & 0.93056 & 0.97955 \\
\hline & 2 & 0.01 & 0.31522 & 0.18231 & 0.17711 & 0.27515 \\
\hline & & 0.05 & 0.47575 & 0.35594 & 0.34942 & 0.46357 \\
\hline \multirow[t]{4}{*}{30} & 1 & 0.01 & 0.91204 & 0.90769 & 0.9025 & 0.97516 \\
\hline & & 0.05 & 0.96727 & 0.96474 & 0.96351 & 0.99141 \\
\hline & 2 & 0.01 & 0.36137 & 0.22570 & 0.21978 & 0.32765 \\
\hline & & 0.05 & 0.53555 & 0.40974 & 0.40636 & 0.5222 \\
\hline \multirow[t]{4}{*}{35} & 1 & 0.01 & 0.94621 & 0.94721 & 0.94446 & 0.98844 \\
\hline & & 0.05 & 0.98264 & 0.98275 & 0.98196 & 0.99635 \\
\hline & 2 & 0.01 & 0.41080 & 0.31898 & 0.26733 & 0.38190 \\
\hline & & 0.05 & 0.5880 & 0.52387 & 0.46557 & 0.57558 \\
\hline \multirow[t]{4}{*}{40} & 1 & 0.01 & 0.96779 & 0.9722 & 0.96801 & 0.99484 \\
\hline & & 0.05 & 0.99112 & 0.99152 & 0.99094 & 0.99851 \\
\hline & 2 & 0.01 & 0.45633 & 0.31898 & 0.30613 & 0.43173 \\
\hline & & 0.05 & 0.63658 & 0.52387 & 0.51386 & 0.62795 \\
\hline \multirow[t]{4}{*}{50} & 1 & 0.01 & 0.98958 & 0.99192 & 0.99104 & 0.99901 \\
\hline & & 0.05 & 0.99767 & 0.99802 & 0.99798 & 0.99976 \\
\hline & 2 & 0.01 & 0.54433 & 0.40077 & 0.39732 & 0.52408 \\
\hline & & 0.05 & 0.71801 & 0.61159 & 0.61013 & 0.71613 \\
\hline \multirow[t]{4}{*}{60} & 1 & 0.01 & 0.99646 & 0.99803 & 0.99754 & 0.99983 \\
\hline & & 0.05 & 0.9994 & 0.99962 & 0.99951 & 0.99999 \\
\hline & 2 & 0.01 & 0.62024 & 0.49343 & 0.48891 & 0.61178 \\
\hline & & 0.05 & 0.78371 & 0.69650 & 0.69528 & 0.78784 \\
\hline
\end{tabular}


Table 4. Power comparison for the tests $\mathrm{CK}_{n}, W^{2}$ and $S^{*}$ when the alternative distribution is $\log$-normal $(\mu, \sigma)$, where $\mu=-0.5,0.5$ and $\frac{E\left(X_{1}^{3}\right)}{3 E\left(X_{1}\right)}=4$ at the significance levels $\alpha=0.01$ and $\alpha=0.05$, and sample sizes $n=5,10,15,20,25,30,35,40,50,60$.

\begin{tabular}{|c|c|c|c|c|c|c|}
\hline$n$ & $\mu$ & $\alpha$ & $\mathrm{CK}_{n}$ & $W^{2}$ & $S^{*}$ & $A^{2}$ \\
\hline \multirow[t]{4}{*}{5} & -0.5 & 0.01 & 0.24482 & 0.1609 & 0.15231 & 0.23526 \\
\hline & & 0.05 & 0.75666 & 0.26966 & 0.26561 & 0.37093 \\
\hline & 0.5 & 0.01 & 0.05227 & 0.02655 & 0.02300 & 0.02989 \\
\hline & & 0.05 & 0.11311 & 0.08119 & 0.07607 & 0.08411 \\
\hline \multirow[t]{4}{*}{10} & -0.5 & 0.01 & 0.4934 & 0.36829 & 0.35331 & 0.47653 \\
\hline & & 0.05 & 0.62451 & 0.52011 & 0.51156 & 0.62469 \\
\hline & 0.5 & 0.01 & 0.12665 & 0.05354 & 0.04532 & 0.06145 \\
\hline & & 0.05 & 0.21189 & 0.13325 & 0.12280 & 0.14577 \\
\hline \multirow[t]{4}{*}{15} & -0.5 & 0.01 & 0.66687 & 0.5464 & 0.53238 & 0.64885 \\
\hline & & 0.05 & 0.78189 & 0.69559 & 0.69094 & 0.77836 \\
\hline & 0.5 & 0.01 & 0.19441 & 0.07906 & 0.06690 & 0.08476 \\
\hline & & 0.05 & 0.30048 & 0.177921 & 0.16629 & 0.19078 \\
\hline \multirow[t]{4}{*}{20} & -0.5 & 0.01 & 0.78654 & 0.69211 & 0.67612 & 0.77269 \\
\hline & & 0.05 & 0.87409 & 0.81428 & 0.80961 & 0.87157 \\
\hline & 0.5 & 0.01 & 0.25970 & 0.10919 & 0.08937 & 0.10978 \\
\hline & & 0.01 & 0.37613 & 0.23036 & 0.21073 & 0.23764 \\
\hline \multirow[t]{4}{*}{25} & -0.5 & 0.01 & 0.86653 & 0.79096 & 0.78446 & 0.86043 \\
\hline & & 0.05 & 0.92845 & 0.88957 & 0.88885 & 0.93059 \\
\hline & 0.5 & 0.01 & 0.31769 & 0.13333 & 0.11645 & 0.13856 \\
\hline & & 0.05 & 0.44050 & 0.27382 & 0.26032 & 0.28537 \\
\hline \multirow[t]{4}{*}{30} & -0.5 & 0.01 & 0.91639 & 0.86450 & 0.85925 & 0.91386 \\
\hline & & 0.05 & 96037 & 0.93594 & 0.93274 & 0.96136 \\
\hline & 0.5 & 0.01 & 0.36862 & 0.16275 & 0.14344 & 0.16947 \\
\hline & & 0.05 & 0.50122 & 0.32030 & 0.30463 & 0.33036 \\
\hline \multirow[t]{4}{*}{35} & -0.5 & 0.01 & 0.94832 & 0.91396 & 0.91064 & 0.94836 \\
\hline & & 0.05 & 0.67824 & 0.96380 & 0.96223 & 0.97858 \\
\hline & 0.5 & 0.01 & 0.41575 & 0.19375 & 0.17400 & 0.19901 \\
\hline & & 0.05 & 0.5120 & 0.36636 & 0.35070 & 0.37294 \\
\hline \multirow[t]{4}{*}{40} & -0.5 & 0.01 & 0.96847 & 0.94655 & 0.94263 & 0.96889 \\
\hline & & 0.05 & 0.98799 & 0.97862 & 0.97822 & 0.98791 \\
\hline & 0.5 & 0.01 & 0.46181 & 0.22860 & 0.19995 & 0.22652 \\
\hline & & 0.05 & 0.59770 & 0.41025 & 0.38660 & 0.41334 \\
\hline \multirow[t]{4}{*}{50} & -0.5 & 0.01 & 0.98915 & 0.98040 & 0.97877 & 0.98985 \\
\hline & & 0.05 & 0.99636 & 0.99345 & 0.99344 & 0.99697 \\
\hline & 0.5 & 0.01 & 0.54406 & 0.28585 & 0.25826 & 0.28815 \\
\hline & & 0.05 & 0.67776 & 0.48859 & 0.46798 & 0.50022 \\
\hline \multirow[t]{4}{*}{60} & -0.5 & 0.01 & 0.99622 & 0.99361 & 0.99314 & 0.99674 \\
\hline & & 0.05 & 0.99899 & 0.99843 & 0.99815 & 0.99924 \\
\hline & 0.5 & 0.01 & 0.61351 & 0.35453 & 0.33283 & 0.35373 \\
\hline & & 0.05 & 0.74134 & 0.56606 & 0.55584 & 0.57902 \\
\hline
\end{tabular}


Table 5. Power comparison for the tests $\mathrm{CK}_{n}, W^{2}$ and $S^{*}$ when the alternative distribution is inverse Gaussian $(\mu, \lambda)$, where $\mu=1,2$ and $\frac{E\left(X_{1}^{3}\right)}{3 E\left(X_{1}\right)}=4$ at the significance levels $\alpha=0.01$ and $\alpha=0.05$, and sample sizes $n=5,10,15,20,25,30,35,40,50,60$.

\begin{tabular}{|c|c|c|c|c|c|c|}
\hline$n$ & $\mu$ & $\alpha$ & $\mathrm{CK}_{n}$ & $W^{2}$ & $S^{*}$ & $A^{2}$ \\
\hline \multirow[t]{4}{*}{5} & 1 & 0.01 & 0.30300 & 0.21251 & 0.20350 & 0.30032 \\
\hline & & 0.05 & 0.41806 & 0.32302 & 0.33007 & 0.44435 \\
\hline & 2 & 0.01 & 0.05933 & 0.02765 & 0.02152 & 0.03139 \\
\hline & & 0.05 & 0.605321 & 0.08506 & 0.07781 & 0.09184 \\
\hline \multirow[t]{4}{*}{10} & 1 & 0.01 & 0.59292 & 0.48651 & 0.47064 & 0.59402 \\
\hline & & 0.05 & 0.71461 & 0.63676 & 0.62848 & 0.72866 \\
\hline & 2 & 0.01 & 0.13676 & 0.05886 & 0.04869 & 0.06677 \\
\hline & & 0.05 & 0.23281 & 0.14449 & 0.13117 & 0.15984 \\
\hline \multirow[t]{4}{*}{15} & 1 & 0.01 & 0.77075 & 0.69261 & 0.67928 & 0.77455 \\
\hline & & 0.05 & 0.86510 & 0.81408 & 0.80707 & 0.86971 \\
\hline & 2 & 0.01 & 0.20769 & 0.09024 & 0.07745 & 0.09663 \\
\hline & & 0.05 & 0.32635 & 0.20271 & 0.18862 & 0.21609 \\
\hline \multirow[t]{4}{*}{20} & 1 & 0.01 & 0.87638 & 0.82720 & 0.81089 & 0.88259 \\
\hline & & 0.05 & 0.93635 & 0.90957 & 0.90308 & 0.94093 \\
\hline & 2 & 0.01 & 0.27532 & 0.12749 & 0.10967 & 0.13040 \\
\hline & & 0.05 & 0.40413 & 0.26343 & 0.24861 & 0.27252 \\
\hline \multirow[t]{4}{*}{25} & 1 & 0.01 & 0.93462 & 0.90622 & 0.89887 & 0.93946 \\
\hline & & 0.05 & 0.96975 & 0.95773 & 0.95509 & 0.97396 \\
\hline & 2 & 0.01 & 0.33726 & 0.16077 & 0.13812 & 0.16702 \\
\hline & & 0.05 & 0.47464 & 0.32183 & 0.29386 & 0.33291 \\
\hline \multirow[t]{4}{*}{30} & 1 & 0.01 & 0.96527 & 0.95098 & 0.94767 & 0.97084 \\
\hline & & 0.05 & 0.98641 & 0.98042 & 0.97945 & 0.98855 \\
\hline & 2 & 0.01 & 0.38750 & 0.19857 & 0.17977 & 0.20348 \\
\hline & & 0.05 & 0.53332 & 0.37252 & 0.36140 & 0.38521 \\
\hline \multirow[t]{4}{*}{35} & 1 & 0.01 & 0.98308 & 0.97402 & 0.97349 & 0.98555 \\
\hline & & 0.05 & 0.99422 & 0.99091 & 0.99068 & 0.99442 \\
\hline & 2 & 0.01 & 0.44343 & 0.23731 & 0.40915 & 0.24297 \\
\hline & & 0.05 & 0.58847 & 0.42793 & 0.21739 & 0.43611 \\
\hline \multirow[t]{4}{*}{40} & 1 & 0.01 & 0.99108 & 0.98823 & 0.98616 & 0.99380 \\
\hline & & 0.05 & 0.9974 & 0.9386 & 0.99593 & 0.99791 \\
\hline & 2 & 0.01 & 0.4908 & 0.28127 & 0.24918 & 0.28369 \\
\hline & & 0.05 & 0.6386 & 0.47844 & 0.46283 & 0.48694 \\
\hline \multirow[t]{4}{*}{50} & 1 & 0.01 & 0.99817 & 0.99738 & 0.99694 & 0.99866 \\
\hline & & 0.05 & 0.99945 & 0.99943 & 0.99330 & 0.99972 \\
\hline & 2 & 0.01 & 0.57869 & 0.35686 & 0.33345 & 0.35936 \\
\hline & & 0.05 & 0.721150 & 0.57137 & 0.55839 & 0.57868 \\
\hline \multirow[t]{4}{*}{60} & 1 & 0.01 & 0.99957 & 0.99951 & 0.99935 & 0.99977 \\
\hline & & 0.05 & 0.99993 & 0.99990 & 0.99990 & 0.99992 \\
\hline & 2 & 0.01 & 0.65200 & 0.44124 & 0.42045 & 0.43945 \\
\hline & & 0.05 & 0.78369 & 0.65516 & 0.64036 & 0.66469 \\
\hline
\end{tabular}




\section{An Illustrative Example}

In this section, we propose the new hypothesis procedure to a practical data set.

Example 2. The data below were given by Caroni (2002), the data on the failure times of 25 ball bearings in endurance test. The $25(=\mathrm{n})$ failure times are $\left\{X_{i}, i=1, \ldots, 25\right\}=17.88,28.92,33.00,41.52,42.12,45.60,48.48,51.84$, $51.96,54.12,55.56,67.80,67.80,67.80,68.64,86.64,68.88,84.12,93.12$, $98.64,105.12,105.84,127.92,128.04,173.40$.

Table 6 shows critical values, test statistics and the $p$-values. Since the values of $C K_{n}$ are less than the critical values, test accepts the null hypothesis that failure times follow a Rayleigh distribution at significance levels $\alpha=0.01$ and $\alpha=0.05$.

Table 6. Critical values, test statistics, and the $p$-values

\begin{tabular}{cccc}
\hline \hline Rayleigh Dis. & Critical value & $\mathrm{CK}_{n}$ & $p$-value \\
\hline$\alpha=0.01$ & 0.5647094 & 0.5120220187 & 0.87849 \\
$\alpha=0.05$ & 0.5421782 & 0.5120220187 & 0.87849 \\
\hline
\end{tabular}

\section{Acknowledgement}

The authors are very grateful to the referee for very helpful comments and suggestions which are incorporated in the paper.

\section{References}

Alizadeh Noughabi, H. and Arghami, N.R. (2011). Monte Carlo Comparison of Five Exponentiality Tests Using Different Entropy Estimates. Journal of Statistical Computation and Simulation, 81, 1579-1592.

Anderson, T.W. and Darling, D.A. (1954). A Test of Goodness-of-Fit. J. Amer. Statist. Assoc., 49, 765-769. 
Asadi, M. and Zohrevand, Y. (2007). On the Dynamic Cumulative Residual Entropy. J. Statist. Plann. Inference, 137, 1931-1941.

Baratpour, S. (2010). Characterizations Based on Cumulative Residual Entropy of First Order Statistics. Commun. Statist. Theor. Meth., 39, 3645-3651.

Baratpour, S. and Habibi Rad, A. (2012). Testing Goodness-of-Fit for Exponential Distribution Based on Cumulative Residual Entropy. Commun. Statist. Theor. Meth., 41, 1387-1396.

Caroni, C. (2002). The Correct Ball Bearing Data. Life-time Data Analysis, 8, 395-399.

Choi, B., Kim, K. and Song, S.H. (2004). Goodness of Fit Test for Exponentiality Based on Kullback-Leibler Information. Commu. Statist. Simul. Computat., 33, 525-537.

Di Crescenzo, A. and Longobardi, M. (2009). On Cumulative Entropies. J. Statist. Plann. Inference, 139, 4072-4087.

Dyer, D.D. and Whisenand, C.W. (1973). Best Linear Estimator of the Parameter of the Rayleigh Distribution-Part I: Small Sample Theory for Censored Order Statistics. IEEE Trans. Reliab., 22, 27-34.

Ebrahimi, N., Soofi, E.S. and Habibullah, M. (1992). Testing Exponentiality Based on Kullback-Leibler Information. Journal of the Royal Statistical Society B, 54, 739-748.

Finkelstein, J. and Schafer, R.E. (1971). Improved Goodness of Fit Tests. Biometrika, 58, 641-645.

Grzegorzewski, P. and Wieczorkowski, R. (1999). Entropy-Based Goodness of Fit Test for Exponentiality. Commun. Statist. Theor. Meth., 28, 1183-1202.

Gurevich, G. and Davidson, A. (2008). Standardized form of Kullback-Leibler Information Based Statistics for Normality and Exponentiality. Computer Modeling and New Technologies, 12, 14-25.

Johnson, N., Kotz, S. and Balakrishnan, N. (1994). Continuous Univariate Distributions. Vol. 1, 2nd ed. Boston, MA: Houghton Mifflin.

Kullback, S. and Leibler, R.A. (1951). On Information and Sufficiency. Annals of Mathematical Statistics, 22, 79-86.

Kullback, S. (1959.) Information Theory and Statistics. John Wiley and Sons, New York.

Kullback, S. (1987). Letter to the Editor: The Kullback-Leibler Distance. The American Statistician, 41, 340-341.

Miller, K. and Sackrowttz, H. (1967). Relationships Between Biased and Unbiased Rayleigh Distributions. SIAM journal on applied Mathematics, 15, 1490-1495. 
Polovko, A.M. (1969). Fundamentals of Reliability Theory. Academic Press, New York.

Rao, M. (2005). More on A New Concept of Entropy and Information. J. Theoret. Probab., 18, 967-981.

Rao, M., Chen, Y., Vemuri, B.C. and Wang, F. (2004). Cumulative Residual Entropy: A New Measure of Information. IEEE Trans. Inform. Theory, 50, 1220-1228.

Shannon, C.E. (1948). A Mathematical Theory of Communication. Bell System Tec. J., 27, $379-432$.

Siddiqui, M.M. (1962). Some Problems Connected with Rayleigh Distribution. Journal of Research of the National Bureau of Standards, 66D, 167-174.

Taufer, E. (2002). On Entropy Based Test for Exponentiality. Commu. Statist. Simul. Computat., 31, 189-200.

Van-Soest, J. (1969). Some Goodness of Fit Tests for the Exponential Distribution. Statistica Neerlandia, 23, 41-51.

Vasicek, O. (1976). A Test for Normality Based on Sample Entropy. J. Royal Statist. Soc. B, 38, 730-737.

Wang, F., Vemuri, B.C., Rao, M. and Chen, Y. (2003). A New and Robust Information Theoretic Measure and Its Application to Image Alignment. Information Processing in Medical Imaging. Lecture Notes in Computer Science, 2732, Springer, Heidelberg, 388-400.

Wang, F. and Vemuri, B.C. (2007). Non-Rigid Multi-Modal Image Registration Using CrossCumulative Residual Entropy. Internat. J. Comput. Vision, 74, 201-215.

\section{S. Baratpour}

Department of Statistics and Ordered and Spatial Data Center of Excellence, Ferdowsi University of Mashhad, Mashhad, Iran.

email: baratpour@um.ac.ir

\section{F. Khodadadi}

Department of Statistics and Ordered and Spatial Data Center of Excellence, Ferdowsi University of Mashhad, Mashhad, Iran.

email: ne_khodadad2@yahoo.com 